\title{
Target refractions in bilateral monofocal pseudophakia: analysis of a mathematical model
}

\author{
William Frith Harris*, Finn Kristian Næser` and Jesper Østergaard Hjortdal§
}

*Department of Optometry, University of Johannesburg, P O Box 524, Auckland Park 2006, South Africa

fDepartment of Ophthalmology, Randers Regional Hospital, 8930 Randers NØ, Denmark

$\S$ Department of Ophthalmology, Aarhus University Hospital, 8000 Aarhus C, Denmark

<wharris@uj.ac.za> <krisnaes@rm.dk> <jesper.hjortdal@dadlnet.dk>

Received 5 May 2013; revised version accepted 27 September 2013

\begin{abstract}
A recent paper proposes a model for suitable target refractions for monofocal pseudophakic monovision. The purpose of this paper is to examine the mathematics that underpins the theory. The expressions for the optimal target refractions presented before are shown formally to define the minimum of the mean binocular refractive error over a range of object distances. The model is shown to be much more sensitive to
\end{abstract}

errors in the distal target refraction than to errors in the proximal refraction. It is also more sensitive to the choice of proximal limit of the object range than to the distal limit. These considerations are important in selecting and evaluating suitable combinations of refractions in pseudophakic monovision. (S Afr Optom 2013 72(3) 103-109)

Key words: Target refraction, pseudophakia, monovision, mathematical model, astigmatism

\section{Introduction}

The aim of contemporary cataract surgery is to obtain optimal uncorrected visual acuity over the most extended fixation range. This may be achieved by the system of monovision where one eye is targeted for distance and the other eye for near. ${ }^{1,2}$ Monovision has been widely used with contact lenses. In younger presbyopes some residual natural lens accommodation remains in eyes fitted with contact lenses and the procedure is essentially reversible. ${ }^{3} \mathrm{On}$ the other hand there is essentially no accommodation in the pseudophakic eye, and any change in refraction will require new surgical procedures ${ }^{4,5}$. It is therefore important to establish appropriate target refractions for the far and near eye, and to analyse the impact of deviations from these targets.

A simple model has recently been described by Naeser and coworkers ${ }^{6}$ for defining suitable target refractions $F_{\mathrm{D}}$ and $F_{\mathrm{P}}$ for each eye following surgery for bilateral monofocal pseudophakia. Subscripts D and $\mathrm{P}$ suggest distal and proximal respectively. The model leads to the optimal target refractions

$F_{\mathrm{Dm}}=\frac{2}{z_{\mathrm{D}}-\sqrt{z_{\mathrm{D}} z_{\mathrm{P}}}}$,

the distal refraction for one eye, and

$F_{\mathrm{Pm}}=\frac{2}{z_{\mathrm{P}}-\sqrt{z_{\mathrm{D}} z_{\mathrm{P}}}}$,

the proximal refraction for the other eye. $z_{\mathrm{D}}$ and $z_{\mathrm{P}}$ are the axial positions defined in Figure 1; they 
represent a range in front of the eye within which object points are supposed to be visible with minimal blur. They are measured in the direction opposite to the direction of light which is defined by longitudinal axis $\mathrm{Z}$ and are negative. We set

$z_{\mathrm{D}}<z_{\mathrm{P}}<0$.

Because hyperopic refractions would induce blur for both distant and proximal fixation we take the target refractions to be myopic and set

$F_{\mathrm{P}}<F_{\mathrm{D}}<0$.

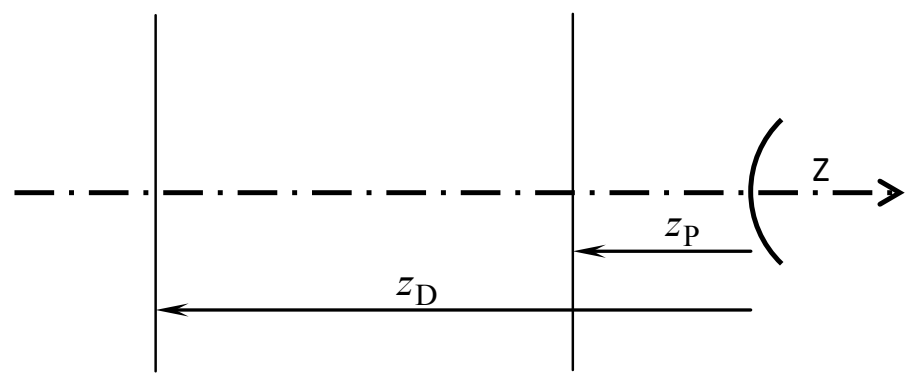

Figure 1 Range of positions in front of the eye in which objects should be seen with minimal blur. $z_{\mathrm{D}}$ and $z_{\mathrm{P}}$ represent the distal and proximal limits of the range. Both are negative.

The purpose of this paper is to take a closer look at the mathematics underlying the theory. We examine the nature of the mean binocular error as a function of the four variables $F_{\mathrm{D}}, F_{\mathrm{P}}, z_{\mathrm{D}}$ and $z_{\mathrm{P}}$ and show formally that Equations 1 and 2 do indeed represent a global minimum. We also examine the nature of the mean binocular error in the neighbourhood of the minimum. It is convenient here to use a mathematical notation which differs from that used originally ${ }^{6}$.

The surgeon's objective is to give the patient optimal vision for objects in the range from a distant transverse plane at axial position $z_{\mathrm{D}}$ to a proximal transverse plane at axial distance $z_{\mathrm{P}}$ (Figure 1). What refractions $F_{\mathrm{D}}$ and $F_{\mathrm{P}}$ should he or she aim for in the two eyes? Figure 1 suggests that $z_{\mathrm{D}}$ and $z_{\mathrm{P}}$ are measured from the cornea; they could also be measured from the spectacle plane. The refractions in those cases are corneal- and spectacle-plane refractions respectively.

\section{Pseudophakic eye}

Implicit in the discussion so far is that the refractions of the two pseudophakic eyes should be stigmatic, that is, $F_{\mathrm{D}}$ and $F_{\mathrm{P}}$ should be real scalars. However there are advocates of astigmatic refractions ${ }^{7-11}$. In order to allow for that possibility we work instead with the $2 \times 2$ dioptric power matrix $\mathbf{F}$ instead of the scalar $F$. Scalar target refractions $F_{\mathrm{D}}$ and $F_{\mathrm{P}}$ are replaced by matrix target refractions $\mathbf{F}_{\mathrm{D}}$ and $\mathbf{F}_{\mathrm{P}}$. As explained elsewhere ${ }^{12} \mathbf{F}$ can be expanded as

$\mathbf{F}=\mathbf{I} F_{\mathrm{I}}+\mathbf{J} F_{\mathrm{J}}+\mathbf{K} F_{\mathrm{K}}$

where $\mathbf{I}$ is an identity matrix,

$\mathbf{J}=\left(\begin{array}{cc}1 & 0 \\ 0 & -1\end{array}\right)$,

$\mathbf{K}=\left(\begin{array}{ll}0 & 1 \\ 1 & 0\end{array}\right)$

and $F_{\mathrm{I}}, F_{\mathrm{J}}$ and $F_{\mathrm{K}}$ are scalar coefficients of the power F . $F_{\text {I }}$ represents the scalar component; it is identical to what Naeser and Hjortdal ${ }^{6,13,14}$ represent by SEP and what is commonly called the equivalent sphere. $F_{\mathrm{J}}$ and $F_{\mathrm{K}}$ account for the astigmatism; they are essentially equivalent to what Naeser and Hjortdal ${ }^{14}$ represent by $\mathrm{KP}(90)$ and $\mathrm{KP}(135)$ and Thibos and coworkers ${ }^{15}$ by $J_{0}$ and $J_{45}$ respectively. The magnitude of the power $\mathbf{F}$ is ${ }^{12}$

$F=\sqrt{F_{\mathrm{I}}^{2}+F_{\mathrm{A}}^{2}}$

where

$F_{\mathrm{A}}=\sqrt{F_{\mathrm{J}}^{2}+F_{\mathrm{K}}^{2}}$

can be thought of as the magnitude of the astigmatism. In particular the coefficients of $\mathbf{F}_{\mathrm{D}}$ are $F_{\mathrm{DI}}, F_{\mathrm{DJ}}$ and $F_{\mathrm{DK}}$ and the two magnitudes are $F_{\mathrm{D}}$ and $F_{\mathrm{DA}}$ and similarly for $\mathbf{F}_{\mathrm{P}}$. Thus the surgeon's objective is rephrased as, for what possibly astigmatic distal and proximal refractions $\mathbf{F}_{\mathrm{D}}$ and $\mathbf{F}_{\mathrm{P}}$ should he or she aim in order to obtain optimal visual acuity in the fixation interval from $z_{\mathrm{D}}$ to $z_{\mathrm{P}}$ ?

Consider a pseudophakic eye of refraction $\mathbf{F}$ which views an object in a transverse plane at axial position $z$. If it so happens that $\mathbf{F}=\mathbf{I} / z$ there will be no blur. In general there is an error

$\mathbf{F}_{\mathrm{e}}=\mathbf{F}-\mathbf{I} / z$

of magnitude

$F_{\mathrm{e}}=\sqrt{\left(F_{\mathrm{I}}-1 / z\right)^{2}+F_{\mathrm{A}}^{2}}$ 
which we take to be a measure of the blur in that eye when it views the object at axial position $z$. (Elsewhere ${ }^{6}$ it is called the defocus.) It is evident from Equation 11 that the error is minimized if $F_{\mathrm{A}}^{2}=0$. This implies $F_{\mathrm{J}}=F_{\mathrm{K}}=0$ in Equation 9 and, hence, that the refraction is $\mathbf{F}=\mathbf{I} F_{\mathrm{I}}$ and, hence, is purely stigmatic. It follows that the refraction can be completely characterized by the scalar $F_{\mathrm{I}}$ and that we can drop matrices from the discussion and revert to scalars. Furthermore the subscript on $F_{\mathrm{I}}$ is redundant and we can write $F$ instead. Thus Equation 11 reduces to

$$
F_{\mathrm{e}}=\sqrt{(F-1 / z)^{2}} .
$$

We shall refer to $F_{\mathrm{e}}$ simply as the (monocular) error.

Below we shall need the integral of the error $F_{\mathrm{e}}$ over the range $z_{\mathrm{D}}$ to $z_{\mathrm{P}}$, namely

$\int_{z_{\mathrm{D}}}^{z_{\mathrm{P}}} F_{\mathrm{e}} d z=\left(z_{\mathrm{P}}+z_{\mathrm{D}}\right) F-\log z_{\mathrm{P}} z_{\mathrm{D}} F^{2}-2$.

(Elsewhere ${ }^{6}$ it is called the cumulative defocus.)

Written in particular for the eye with refraction $F_{\mathrm{D}}$ Equation 12 becomes

$$
F_{\mathrm{De}}=\sqrt{\left(F_{\mathrm{D}}-1 / z\right)^{2}}
$$

and similarly for the eye with refraction $F_{\mathrm{P}}$ and error $F_{\mathrm{Pe}}$. The two errors $F_{\mathrm{De}}$ (blue) and $F_{\mathrm{Pe}}$ (red) vary with $z$ as illustrated in Figure 2. Figure 2 is drawn for $F_{\mathrm{P}}=-0.27 \mathrm{D}$ and $F_{\mathrm{P}}=-1.15 \mathrm{D}$, the refractions calculated before ${ }^{6}$. The two error curves intersect in the point with axial position $z_{\chi}$ and error $F_{\mathrm{e} \chi}$. Hence, from Equation 14 and its counterpart for $F_{\mathrm{Pe}}$, we obtain

$\sqrt{\left(F_{\mathrm{D}}-1 / z_{\chi}\right)^{2}}=\sqrt{\left(F_{\mathrm{P}}-1 / z_{\chi}\right)^{2}}$

or

$$
F_{\mathrm{D}}-1 / z_{\chi}= \pm\left(F_{\mathrm{P}}-1 / z_{\chi}\right) \text {. }
$$

The plus sign corresponds to the case $F_{\mathrm{D}}=F_{\mathrm{P}}$ which is disallowed by Inequality 4 . We are left with

$$
F_{\mathrm{P}}-\frac{1}{z_{\chi}}=-F_{\mathrm{D}}+\frac{1}{z_{\chi}}
$$

and, hence,

$$
z_{\chi}=\frac{2}{F_{\mathrm{D}}+F_{\mathrm{P}}}
$$

or

$$
\frac{1}{z_{\chi}}=\frac{F_{\mathrm{D}}+F_{\mathrm{P}}}{2}
$$

Thus the two error curves intersect where the inverse of the axial position is simply the arithmetic average of the two refractions.

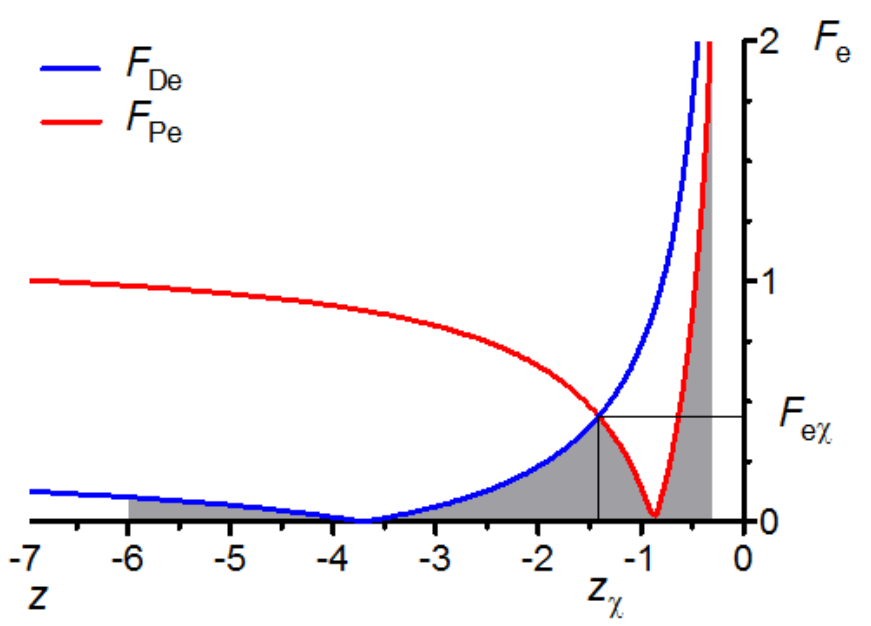

Figure 2 The monocular errors $F_{\mathrm{De}}$ (blue) and $F_{\mathrm{Pe}}$ (red) for refractions $F_{\mathrm{D}}=-0.27 \mathrm{D}$ and $F_{\mathrm{P}}=-1.15 \mathrm{D}$. (Modified from Reference 6.)

Substitution from Equation 19 into Equation 14 gives the error at the intersection,

$F_{\mathrm{e} \chi}=\frac{F_{\mathrm{D}}-F_{\mathrm{P}}}{2}$.

Thus the inverse of the axial position of the intersection in Figure 2 is the semi-sum (Equation 19) and the error there is the semi-difference (Equation 20) of the two refractions. In particular for Figure $2 \frac{1}{z_{\chi}}=-0.17 \mathrm{D}$ and $F_{\mathrm{e} \chi}=0.44 \mathrm{D}$ approximately.

\section{Binocular error}

In agreement with the assumption made by Naeser and coworkers ${ }^{6}$ we now define the binocular error $F_{\text {be }}$ 
as the lower of the two monocular errors. Thus

$F_{\mathrm{be}}=\left\{\begin{array}{l}F_{\mathrm{De}} \text { for } z \leq z_{\chi} \\ F_{\mathrm{Pe}} \text { for } z>z_{\chi}\end{array}\right.$,

as is clear from Figure 2. The mean binocular error over the range of object positions from $z_{\mathrm{D}}$ to $z_{\mathrm{P}}$ is

$\bar{F}_{\text {be }}=\frac{1}{z_{\mathrm{P}}-z_{\mathrm{D}}} \int_{z_{\mathrm{D}}}^{z_{\mathrm{P}}} F_{\mathrm{be}} d z$

which, making use of Equation 21, we can split into the sum of two integrals as

$\bar{F}_{\text {be }}=\frac{1}{z_{\mathrm{P}}-z_{\mathrm{D}}}\left(\int_{z_{\mathrm{D}}}^{z_{\chi}} F_{\mathrm{De}} \mathrm{dz}+\int_{z_{\chi}}^{z_{\mathrm{P}}} F_{\mathrm{Pe}} \mathrm{dz}\right)$

Each integral is of the form of Equation 13. Hence, applying Equation 13 twice we obtain

$$
\begin{gathered}
\bar{F}_{\text {be }}=\frac{1}{z_{\mathrm{P}}-z_{\mathrm{D}}}\left(\left(z_{\chi}+z_{\mathrm{D}}\right) F_{\mathrm{D}}-\log z_{\chi} z_{\mathrm{D}} F_{\mathrm{D}}^{2}-2 .\right. \\
\left.+\left(z_{\mathrm{P}}+z_{\chi}\right) F_{\mathrm{P}}-\log z_{\mathrm{P}} z_{\chi} F_{\mathrm{P}}^{2}-2\right)
\end{gathered}
$$

which with substitution from Equation 18 simplifies to

$$
\bar{F}_{\mathrm{be}}=\frac{1}{z_{\mathrm{P}}-z_{\mathrm{D}}}\left(z_{\mathrm{D}} F_{\mathrm{D}}+z_{\mathrm{P}} F_{\mathrm{P}}+\log \frac{\left(\frac{1}{F_{\mathrm{P}}}+\frac{1}{F_{\mathrm{D}}}\right)^{2}}{4 z_{\mathrm{P}} z_{\mathrm{D}}}-2\right) .
$$

Equation 25 represents the mean binocular error as a function of the four independent variables, the two refractions $F_{\mathrm{D}}$ and $F_{\mathrm{P}}$ and the extremes of the object range $z_{\mathrm{D}}$ and $z_{\mathrm{P}}$.

Equation 25 is plotted as a surface in Figure 3 for $z_{\mathrm{D}}=-6 \mathrm{~m}$ and $z_{\mathrm{P}}=-0.33 \mathrm{~m}$ for a range of values of $F_{\mathrm{P}}$ and a range of values of $F_{\mathrm{D}}$. The height of the surface represents the mean binocular error $\bar{F}_{\text {be }}$ for the particular values of $z_{\mathrm{D}}, z_{\mathrm{P}}, F_{\mathrm{D}}$ and $F_{\mathrm{P}}$. Figure 4 is a contour plot of the surface. The dot locates the minimum mean binocular error $\bar{F}_{\text {bem }}=0.1712$ D. The contours are at intervals of $0.025 \mathrm{D}$. The innermost contour is at $0.175 \mathrm{D}$. Towards the top left the surface rises steeply; contours there at levels greater than $0.5 \mathrm{D}$ have been omitted. As these figures show the surface has the form of a relatively long and narrow valley with a single minimum.

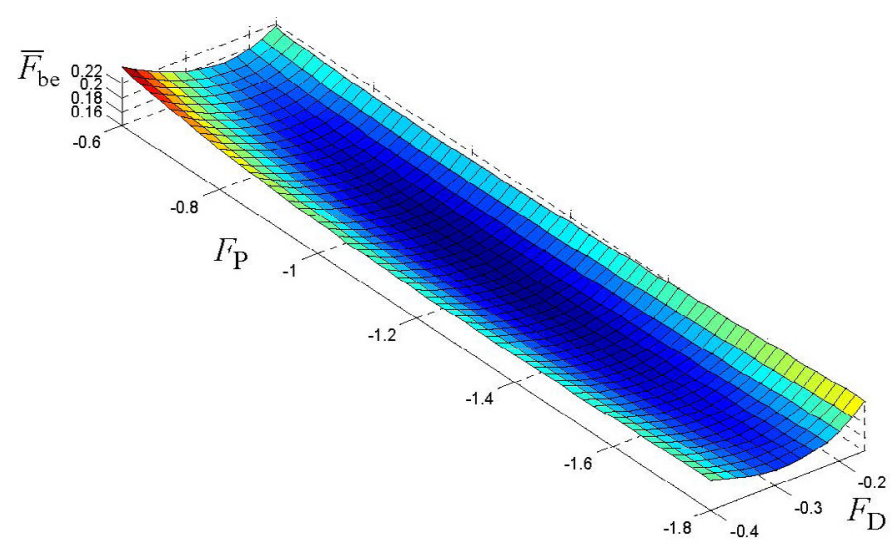

Figure 3 The mean binocular error $\overline{F_{\text {be }}}$ as a function of the proximal $F_{\mathrm{P}}$ and distal $F_{\mathrm{D}}$ refractions for a region around the minimum, the units being dioptres. The object range is $z_{\mathrm{D}}=-6 \mathrm{~m}$ to $z_{\mathrm{P}}=-0.33 \mathrm{~m}$.

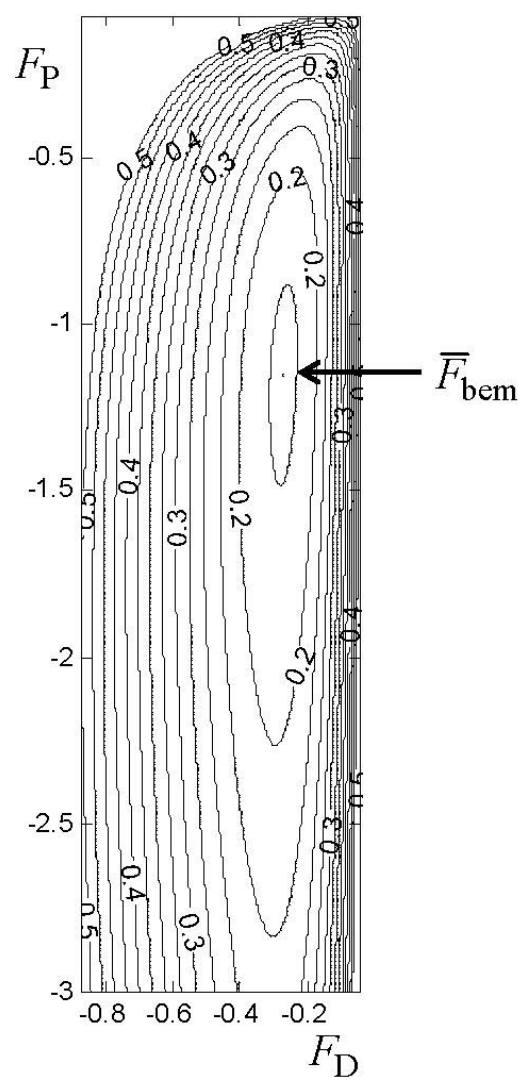

Figure 4 A contour plot of the mean binocular error $\bar{F}_{\text {be }}$ as a function of the proximal $F_{\mathrm{P}}$ and distal $F_{\mathrm{D}}$ refractions for the object range $z_{\mathrm{D}}=-6$ to $z_{\mathrm{P}}=-0.3 \mathrm{~m}$. The minimum mean binocular error, $\bar{F}_{\text {bem }}=0.1712 \mathrm{D}$, is represented by the dot, the corresponding optimum target refractions being $F_{\mathrm{Pm}}=-0.1513$ and $F_{\mathrm{Dm}}=-0.2700 \mathrm{D}$. The contours are at intervals of $0.025 \mathrm{D}$. The innermost contour is at $0.175 \mathrm{D}$. Towards the top left the surface rises steeply; contours there at levels greater than $0.5 \mathrm{D}$ have been omitted. 


\section{Dependence of the mean binocular error on the two refractions}

From Equation 25 we obtain the two derivatives

$\frac{\partial \bar{F}_{\mathrm{be}}}{\partial F_{\mathrm{D}}}=\frac{1}{z_{\mathrm{P}}-z_{\mathrm{D}}}\left(z_{\mathrm{D}}-\frac{2}{F_{\mathrm{D}}\left(\frac{F_{\mathrm{D}}}{F_{\mathrm{P}}}+1\right)}\right)$

and

$$
\frac{\partial \bar{F}_{\mathrm{be}}}{\partial F_{\mathrm{P}}}=\frac{1}{z_{\mathrm{P}}-z_{\mathrm{D}}}\left(z_{\mathrm{P}}-\frac{2}{F_{\mathrm{P}}\left(\frac{F_{\mathrm{P}}}{F_{\mathrm{D}}}+1\right)}\right) .
$$

We are seeking values of refractions $F_{\mathrm{D}}$ and $F_{\mathrm{P}}$ that minimize the mean binocular error $\bar{F}_{\text {be }}$ (given by Equation 25) over a specified object range from $z_{\mathrm{D}}$ to $z_{\mathrm{P}}$. They are the optimal target refractions which we represent by $F_{\mathrm{Dm}}$ and $F_{\mathrm{Pm}}$. A necessary condition that $\bar{F}_{\text {be }}$ has a minimum at $F_{\mathrm{D}}=F_{\mathrm{Dm}}$ and $F_{\mathrm{P}}=F_{\mathrm{Pm}}$ is that the derivatives of Equations 26 and 27 vanish. This gives us two equations in the two unknowns $F_{\mathrm{Dm}}$ and $F_{\mathrm{Pm}}$. Solving them we find that

$$
F_{\mathrm{Dm}}=\frac{2}{z_{\mathrm{D}} \pm \sqrt{z_{\mathrm{D}} z_{\mathrm{P}}}}
$$

and

$$
F_{\mathrm{Pm}}=\frac{2}{z_{\mathrm{P}} \pm \sqrt{z_{\mathrm{D}} z_{\mathrm{P}}}}
$$

Inequality 3 implies that $\sqrt{z_{\mathrm{D}} z_{\mathrm{P}}}>\left|z_{\mathrm{P}}\right|$. The plus sign in the above two equations then implies $F_{\mathrm{Pm}}>0$ in violation of Inequality 4 and must, therefore, be dropped from both equations. Hence we obtain Equations 1 and 2.

Equations 1 and 2 are necessary but not sufficient conditions for a minimum in binocular error. To ensure they represent a minimum we need to determine second derivatives. They turn out to be

$$
\frac{\partial^{2} \bar{F}_{\mathrm{be}}}{\partial F_{\mathrm{D}}^{2}}=\frac{2 F_{\mathrm{P}}\left(2 F_{\mathrm{D}}+F_{\mathrm{P}}\right)}{\left(z_{\mathrm{P}}-z_{\mathrm{D}}\right)\left(F_{\mathrm{D}}+F_{\mathrm{P}}\right)^{2} F_{\mathrm{D}}^{2}}
$$

$\frac{\partial^{2} \bar{F}_{\mathrm{be}}}{\partial F_{\mathrm{P}}^{2}}=\frac{2 F_{\mathrm{D}}\left(2 F_{\mathrm{P}}+F_{\mathrm{D}}\right)}{\left(z_{\mathrm{P}}-z_{\mathrm{D}}\right)\left(F_{\mathrm{P}}+F_{\mathrm{D}}\right)^{2}{F_{\mathrm{P}}}^{2}}$

and

$\frac{\partial^{2} \bar{F}_{\mathrm{be}}}{\partial F_{\mathrm{P}} \partial F_{\mathrm{D}}}=-\frac{2}{\left(z_{\mathrm{P}}-z_{\mathrm{D}}\right)\left(F_{\mathrm{D}}+F_{\mathrm{P}}\right)^{2}}$.

Then

$$
\begin{gathered}
\frac{\partial^{2} \bar{F}_{\mathrm{be}}}{\partial F_{\mathrm{D}}^{2}} \frac{\partial^{2} \bar{F}_{\mathrm{be}}}{\partial F_{\mathrm{P}}^{2}}-\left(\frac{\partial^{2} \bar{F}_{\mathrm{be}}}{\partial F_{\mathrm{P}} \partial F_{\mathrm{D}}}\right)^{2} \\
=\frac{8}{F_{\mathrm{D}} F_{\mathrm{P}}\left(z_{\mathrm{P}}-z_{\mathrm{D}}\right)^{2}\left(F_{\mathrm{D}}+F_{\mathrm{P}}\right)^{2}} .
\end{gathered}
$$

Because of Inequalities 3 and 4 the right-hand sides of Equations 30, 31 and 33 are all greater than zero. Thus the sufficient condition ${ }^{16}$ is satisfied and, hence, Equations 1 and 2 do indeed define a unique minimum mean binocular error $\bar{F}_{\text {bem }}$ over the specified range of object positions.

Evaluated at the minimum of surfaces of the type shown in Figures 3 and 4 Equations 30 and 31 give the curvature of the surface at the minimum in a direction parallel to the $F_{\mathrm{D}}$ and $F_{\mathrm{P}}$ axes respectively. (A positive curvature means that moving along the surface from the minimum takes one upward; a negative curvature would imply a maximum instead of the minimum we have here.)

For $z_{\mathrm{D}}=-6 \mathrm{~m}$ and $z_{\mathrm{P}}=-0.33 \mathrm{~m}$ Equations 1 and 2 give optimum refractions $F_{\mathrm{Dm}}=-0.2700 \mathrm{D}$ and $F_{\mathrm{Pm}}=-1.1513 \mathrm{D}$. The binocular error is zero at axial distances $1 / F_{\mathrm{Dm}}=-3.7036 \mathrm{~m}$ and $1 / F_{\mathrm{Pm}}=-0.8686 \mathrm{~m}$. For objects at axial distances $z_{\mathrm{D}}=-6 \mathrm{~m}$ and $z_{\mathrm{P}}=-0.33 \mathrm{~m}$ the binocular errors are $0.1033 \mathrm{D}$ and $1.8790 \mathrm{D}$ respectively. The latter figure represents the maximum binocular error for the object range and is at the proximal point of the range. With these optimal refractions the axial position for error equality of the two eyes is (Equation 18) $z_{\chi}=-1.4071 \mathrm{~m}$, the binocular error there being (Equation 14) $F_{\chi}=0.4407 \mathrm{D}$. The mean binocular error for the object range (Equation 25) is $\bar{F}_{\text {be }}=0.1712$ D. With these refractions Equations 30 and 31 result in $\partial^{2} \bar{F}_{\text {be }} / \partial F_{\mathrm{D}}^{2}=4.6636 \mathrm{~m}$ and $\partial^{2} \bar{F}_{\text {be }} / \partial F_{\mathrm{P}}^{2}=0.0915$ 
m. Thus the curvature at the minimum in the $F_{\mathrm{D}}$ direction is more than 50 times the curvature in the $F_{\mathrm{P}}$ direction. There is, therefore, much greater tolerance for errors in the proximal target refraction $F_{\mathrm{Pm}}$ than in the distal target refraction $F_{\mathrm{Dm}}$.

\section{Dependence of the mean binocular error on the object range}

From Equation 25 we also obtain the derivatives

$\frac{\partial \bar{F}_{\mathrm{be}}}{\partial z_{\mathrm{D}}}=\frac{1}{\left(z_{\mathrm{P}}-z_{\mathrm{D}}\right)^{2}}\left(z_{\mathrm{P}}\left(F_{\mathrm{P}}+F_{\mathrm{D}}-\frac{1}{z_{\mathrm{D}}}\right)+\log \frac{\left(\frac{1}{F_{\mathrm{P}}}+\frac{1}{F_{\mathrm{D}}}\right)^{2}}{4 z_{\mathrm{P}} z_{\mathrm{D}}}-1\right)$

and

$\frac{\partial \bar{F}_{\mathrm{be}}}{\partial z_{\mathrm{P}}}=-\frac{1}{\left(z_{\mathrm{P}}-z_{\mathrm{D}}\right)^{2}}\left(z_{\mathrm{D}}\left(F_{\mathrm{P}}+F_{\mathrm{D}}-\frac{1}{z_{\mathrm{P}}}\right)+\log \frac{\left(\frac{1}{F_{\mathrm{P}}}+\frac{1}{F_{\mathrm{D}}}\right)^{2}}{4 z_{\mathrm{P}} z_{\mathrm{D}}}-1\right)$.

For the object range $z_{\mathrm{D}}=-6 \mathrm{~m}$ to $z_{\mathrm{P}}=-0.33 \mathrm{~m}$ and the optimal refractions calculated above one obtains $\partial \bar{F}_{\mathrm{be}} / \partial z_{\mathrm{D}}=0.01196 \mathrm{D} / \mathrm{m} \quad$ and $\partial \bar{F}_{\mathrm{be}} / \partial z_{\mathrm{p}}=0.30120 \mathrm{D} / \mathrm{m}$. Thus the sensitivity of the mean binocular error to small change in the proximal limit of the object range is nearly 30 times that in the distal limit.

\section{Concluding remarks}

The analysis here provides formal proof of the result presented before ${ }^{6}$ that the optimal target refractions $F_{\mathrm{Dm}}$ and $F_{\mathrm{Pm}}$ for monofocal pseudophakia over an object range from $z_{\mathrm{D}}$ to $z_{\mathrm{P}}$ are given by Equations 1 and 2. The optimal target refractions locate the minimum of the mean binocular error over the object range for distal $F_{\mathrm{D}}$ and proximal $F_{\mathrm{P}}$ target refractions. Figures 3 to 4 show the mean binocular error graphically for the object range from $z_{\mathrm{D}}=-6$ to $z_{\mathrm{P}}=-0.33 \mathrm{~m}$. The surface is a long narrow valley with a minimum at the optimal refractions $F_{\mathrm{Dm}}=-0.2700 \mathrm{D}$ and $F_{\mathrm{Pm}}=-1.1513$ D. Because the curvature at the bottom of the valley in the $F_{\mathrm{D}}$ direction is some 50 times the curvature in the $F_{\mathrm{P}}$ direction it means that a small change in distal refraction from the optimum takes one up the sides of the valley much faster than does a small change in proximal refraction. This suggests that errors in the distal refraction are considerably more important than errors in the proximal refraction. Thus small deviations of the proximal refraction from the target refraction have limited impact on the mean binocular error while there may be a need for additional surgery where distal refractions are off target. On the other hand, around the optimum, the mean binocular error is some 30 times more sensitive to the proximal limit $z_{\mathrm{P}}$ of the axial range than to the distal limit $z_{\mathrm{D}}$. Thus the choice of the proximal limit is more important than the choice of the distal limit. It may be important, therefore, to tailor the proximal target for the individual patient.

\section{Acknowledgements}

WFH acknowledges support from the National Research Foundation of South Africa and FKN acknowledges support from the Public Health authorities, Region Midt, of Denmark.

\section{References}

1. Evans BJW. Monovision: a review. Ophthal Physiol Opt 200727 417-439.

2. Johannsdottir KR, Stelmach LB. Monovision: a review of the scientific literature. Optom Vis Sci 200178 646-651.

3. Gutkowski M, Cassin B. Stereopsis and monovision in the contact lens management of presbyopia. Bin Vis $Q 19906$ 31-36.

4. Finkelman YM, Ng JQ, Barrett GD. Patient satisfaction and visual function after pseudophakic monovision. J Cataract Refract Surg 200935 998-1002.

5. Greenbaum S. Monovision pseudophakia. J Cataract Refract Surg 200228 1439-1443.

6. Naeser K, Hjortdal J, Harris WF. Pseudophakic monovision: optimal distribution of refractions. Acta Ophthalmol 2013 May 7. doi: 10.1111/aos.12148. [Epub ahead of print]

7. Huber C. Planned myopic astigmatism as a substitute for accommodation in peudophakia. Am Intraoc Implant Soc $J$ $19817244-249$.

8. Datiles MB, Gancayco T. Low myopia with low astigmatism correction gives cataract surgery patients good depth of focus. Ophthalmol 199097 922-926.

9. Sawusch MR, Guyton DL. Optimal astigmatism to enhance 
depth of focus after cataract surgery. Ophthalmol 199198 $1025-1029$.

10. Bradbury JA, Hilman JS, Cassells-Brown A. Optimal postoperative refraction for good unaided near and distance vision with monofocal intraocular lenses. Br J Ophthalmol 199276 300-302.

11. Trindade F, Oliveira A, Frasson M. Benefit of against-therule astigmatism to uncorrected near acuity. $J$ Cataract Refract Surg 199723 82-85.

12. Harris WF. Optimal target refraction for implantation of monofocal intraocular lenses. Acta Ophthalmol $20129075-$ 76.

13. Naeser K, Hjortdal, J. Optimal refraction with monofocal intraocular lenses: no beneficial effects of astigmatism. Acta Ophthalmol 201189 111-115.

14. Naeser K, Hjortdal, J. Multivariate analysis of refractive data: mathematics and statistics of spherocylinders. $J$ Cataract Refract Surg 200127 129-142.

15. Thibos LN, Wheeler W, Horner D. Power: an application of Fourier analysis to the description and analysis of refractive error. Optom Vis Sci 199774 367-375.

16. Marsden JE, Tromba AJ. Vector Calculus. 3rd ed. Freeman, New York, 1988 p 256. 